\title{
Formation of the control signals based on application of the neural network approaches in spine rehabilitation systems
}

\author{
Nikolay V. Dorofeev*1, Anastasia V. Grecheneva² and Oleg R. Kuzichkin² \\ ${ }^{1}$ Vladimir State University named after A. G. and N. G. Stoletovs, 87 Gorkiy St., 600000, Vladimir, Russia \\ ${ }^{2}$ Belgorod National Research University, 85 Pobedy St., 308015 Belgorod, Russia
}

\section{ABSTRACT}

The article substantiates the necessity of correction of control signals depending on the state of the spine and the movements performed. The generalized structural scheme of the control unit of executive mechanisms in spine rehabilitation systems is considered. As a result of the operation of the control unit, motor exercises are corrected in rehabilitation techniques based on the results of modeling the permissible degree of flexure of the spine. An example of patterns of motor actions is given and a generalized model of motion patterns is described.

\section{KEY WORDS: DIAGNOSTIC, REHABILITATION, NERVOUS SYSTEM, GONIOMETRY, MUSCULOSKELETAL SYSTEM, NEURAL NETWORK}

\section{INTRODUCTION}

The efficiency of modern technical means makes it possible to implement algorithms for real- time processing large amounts information. It is lead to increase the efficiency and quality of medical systems, in particular, rehabilitation systems of the spine. The complexity of developing of this class systems is associated with a greater risk of harm to the health of the patient due to an incorrect diagnosis. This risk also includes erroneous decisions of the rehabilitation system that are incompatible with the life of the patient. The need for adaptation to the various physiological parameters of the patient taking into account of injuries to the spine causes even greater difficulties, (Sobolev et al., 2017 Kulik, 2017).

The aim of the present work is to improve the quality of management of rehabilitation exoskeletons due to the use of neural network algorithms for estimating the permissible degree of flexure of the yesterday.

\section{ARTICLE INFORMATION:}

*Corresponding Author: russia@prescopus.com Received 12 $2^{\text {th }}$ July, 2018

Accepted after revision $23^{\text {rd }}$ Sep, 2018

BBRC Print ISSN: 0974-6455

Online ISSN: 2321-4007 CODEN: USA BBRCBA

\%.: Thomson Reuters ISI ESC / Clarivate Analytics USA and

: Crossref Indexed Journal

NAAS Journal Score 2018: 4.31 SJIF 2017: 4.196

- A Society of Science and Nature Publication, Bhopal India 2018. All rights reserved.

Online Contents Available at: http//www.bbrc.in/

DOI: $10.21786 / \mathrm{bbrc} / 11.3 / 3$ 


\section{THE PROBLEM OF MANAGEMENT OF THE REHABILITATION SYSTEMS}

Currently, the functionality of existing rehabilitation systems is insufficient for patients with spine pathologies. This is due, first of all, to their limited application in conditions of low mobility of the patient (fractures, gunshot wounds, etc.) or the lack of a priori information on the patient's permissible movements (during rehabilitation) without causing additional harm to his health. However, the process of rehabilitation is accelerated if the correct load on the pathological parts of the spine is calculated (Yezhov et al., 2013; Tuktamyshev Et Bezmaternykh, 2014; Vengerova \&t Solovyova, 2008; Zubareva, 2011, Maksimova, 2012; Kulik, 2017, Sobolev et al., 2017).

The control signals for the rehabilitation exoskeleton are formed on the basis of the patient's desired movements and are limited by the physiological parameters and state of the patient.

Arbitrary movements of the patient are formed by the exoskeleton on the basis of the recorded nerve (electroencephalography), muscle (electromyography). Involuntary movements of the patient are formed by the mechanical (strain gage) signals of the exoskeleton at different stages of motor processes in various (informative) areas of the patient's body, (Grecheneva et al., 2017).

Problems in recording arbitrary movements of the patient are the error of the measuring path, the quality of recognition of informative signals and pathology of the human neuromuscular system.

So, for example, all movements of the musculoskeletal system of a human without pathologies begin in the central nervous system, namely in the motor zone of the cerebral cortex. The generated electrical signals of movement (motion impulses) from the brain through the spinal cord are transmitted to the peripheral nervous system along those nerve fibers (motor neurons) that must cause the necessary contractions of the muscular system, (Sobolev et al., 2017).

Motor neurons have feedbacks, which receive information from muscle fibers, receptors and other sensory receptors, in order to further coordinate movement and prevent muscle damage. Since the moment of formation of an impulse in the cerebral cortex before the movement (contraction or relaxation of the muscles), some time passes, individual intervals of which are described in (Sinitskaya \&t Gribanov, 2014; Zakharova et al., 2012; Grecheneva et al., 2017).

In general, the movement (especially arbitrary) is the result of complex neuropsychophysiological processes in which a plan of motion or reaction to stimuli is formed, and its constant correction occurs throughout the entire movement. In addition to the motor zone of the cerebral cortex, other areas of the brain are involved: the posterior parietal cortex, the limbic system, the cerebellum, the frontal cortex, etc. (Sinitskaya \&t Gribanov, 2014).

When processing and analyzing the signals of motor neuron activity, attention should be paid to the fact that useful signals, although cyclic, are not stationary. In addition, the distribution of the noise component of the signals is not normal (Zakharova et al., 2012).

Functional changes of any part of the path from the place of formation of motor signals to the muscle cause changes in the parameters of motion of the involved kinematic pairs and the musculoskeletal system as a whole. Figure 1 shows the averaged electromyograms obtained (Fig. 2a and 2b) and the dynamics of the deviation angle from the axis of the spine (Fig. 2c and $2 \mathrm{~d}$ ) in the state of rest of a healthy person (Fig. 2a and 2c) and a person with a tremor of the back muscles (Fig. $2 \mathrm{~b}$ and 2d) (Butukhanov, 2009).

Deviations in the electrophysiological signals involved in the locomotion activity of the musculoskeletal system, from normal values for healthy people manifest themselves in amplitude, phase, shape, and other characteristics of the signals and depend on the different concentration of the attention, the accuracy etc. (Voznesenskaya, 2006; Doronin \& Doronina, 2010; Rakhmilevich et al., 2012; Efımov, 2012, Zakharova \&t Shemirova, 2016; Shchenyavskaya \&t Zakharova, 2015; Zakharova et al., 2016).

The need for high accuracy of recording of the patient movements is due to possible damage to nerve fibers and the nervous system as a whole. When a nerve tissue is damaged, a number of processes occur successively, leading to the death of damaged nerve cells and the subsequent death of intact ones. According to modern ideas, the main factors leading to the destruction of nerve cells are a violation of microcirculation, hypoxia and ischemia. There is a link between the degree of neuronal damage and the change in the level of the constant potential and the membrane potential of neurons (Sufianova and Shapkin 2014; Shanitsin et al., 2013).

Damage to the nervous tissues of the spinal cord changes the frequency and amplitude of the spinal cord signals and depends on the amount of pressure (compression) and the degree of damage to the nerve fiber. Relying on the works on a dependence of the amplitude of the electrospinogram on the subdural pressure can be described in accordance with Table 1.

Thus, damage to the spinal cord causes an increase in spontaneous electrical activity, and in case of significant damage, further decrease in spontaneous electrical activity. In this case, the frequency characteristics of the activity of the spinal cord correspond to the frequency characteristics of activity of the cerebral cortex, but with 


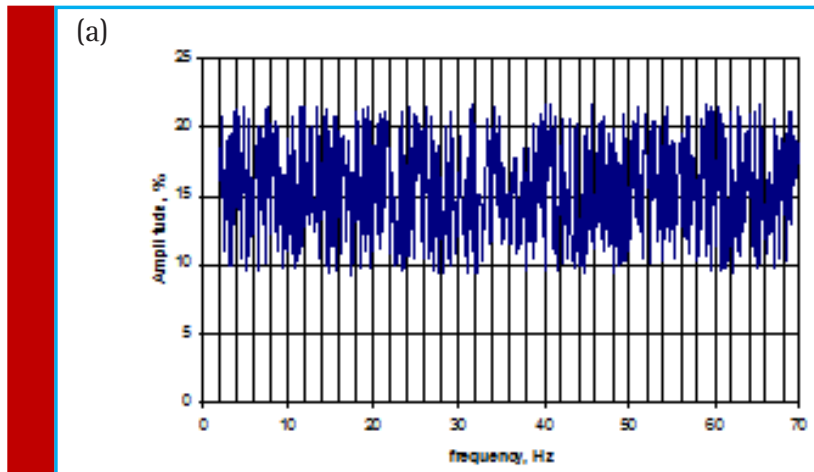

(b)

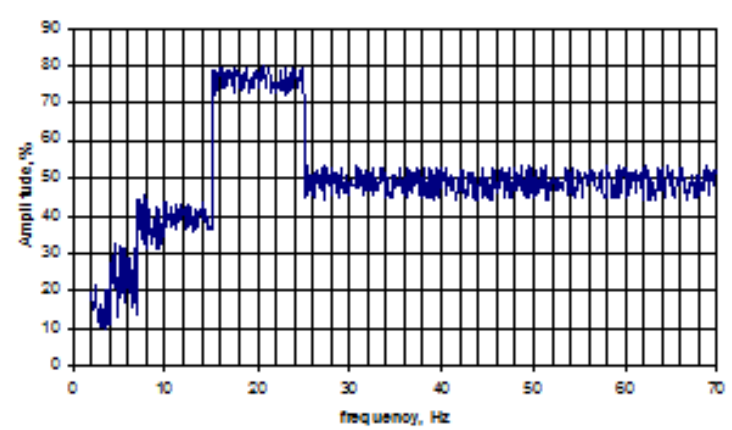

(c)

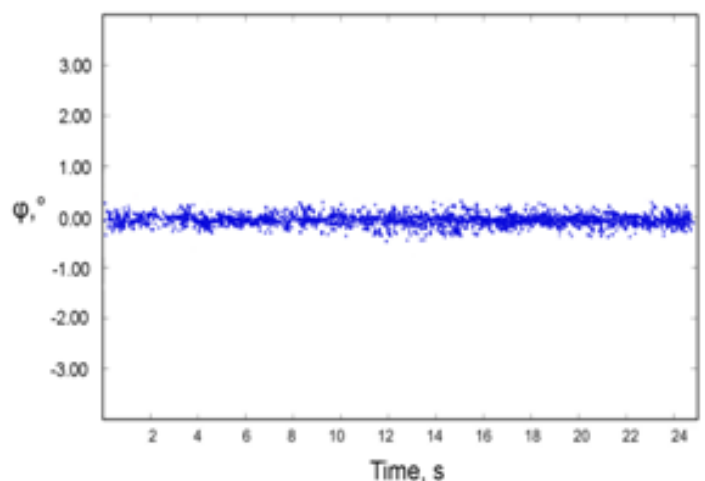

(d)

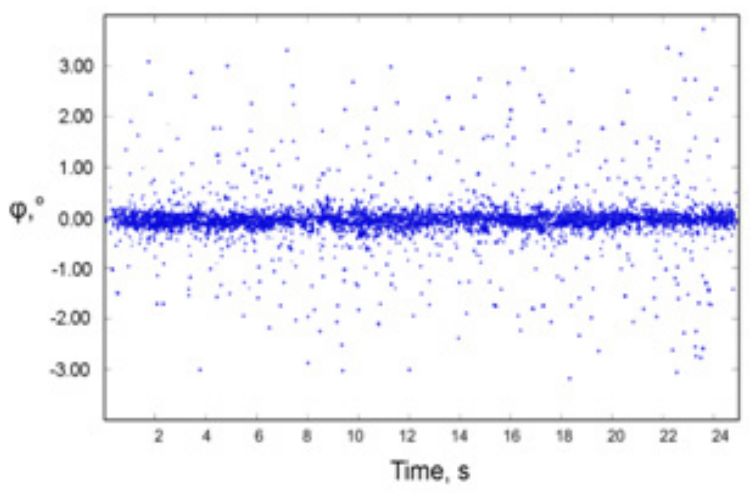

FIGURE 1. Recording of the averaged electromyogram $(\mathrm{a}, \mathrm{b})$ and the dynamics of the deviation angle from the axis of the spine in the state of rest of a healthy person (c) and a patient with a tremor of back muscles (d)

smaller amplitude (in the spinal cord). A rupture of the spinal cord increases the level of the constant potential and lowers the values on the electro-spinogram of segments lying below the trauma, and leads to an increase in electrophysiological changes as the distance decreases from damage.

Segments lying above the damage zone are characterized by a decrease in the level of the constant potential and the total amplitude on the electrospinograms, a decrease in the magnitude of electrophysiological deviations. With pressure on the spinal cord, the level of the constant potential shifts and the amplitude decreases on the electrospinograms. The degree of violations in signals decreases is removed from the site of pressure. When the pressure on the spinal cord decreases, repolarization occurs and the amplitude increases again on the electrospinograms. A complete restoration of the level of constant potential does not occur. Thus, the complex processing of the values of the level of the constant potential and electric activity of the spinal cord makes it possible to evaluate the electrophysiological violations and functional changes in the spinal cord both in the injury zone and in neighboring areas (Kulik, 2017).

Thus, the formation of control signals for the rehabilitation exoskeleton is determined not only by the chosen recovery technique, but also by the patient's vertebral state (vertebral, interarticulate fluid, interarticulate cartilage and neural fiber regions).

\section{CONTROL UNIT FOR REHABILITATION EXOSKELETON}

The task of the control unit for the rehabilitation exoskeleton is to generate control signals for the actuators (Figure 2).

The control unit consists of two functional parts: a forecast generation unit and a decision block. The forecasting unit evaluates the location, extent and likelihood of damage to the bony, cartilaginous and nerve tissues of the spine. The prognostic estimation is formed on the

\begin{tabular}{|l|l|l|l|l|l|l|l|l|l|}
\hline Table 1. Dependence of the amplitude of the electrospinogram from subdural pressure \\
\hline Pressure, $\mathrm{mm} . \mathrm{gt} ;$ & 183 & 250 & 300 & 350 & 400 & 450 & 500 & 550 & 560 \\
\hline Amplitude, $\mu \mathrm{V}$ & $31 \pm 9$ & $40 \pm 10$ & $52 \pm 12$ & $54,5 \pm 15,5$ & $64 \pm 18$ & $78 \pm 20$ & $82,5 \pm 15,5$ & $73 \pm 15$ & $68 \pm 15$ \\
\hline
\end{tabular}




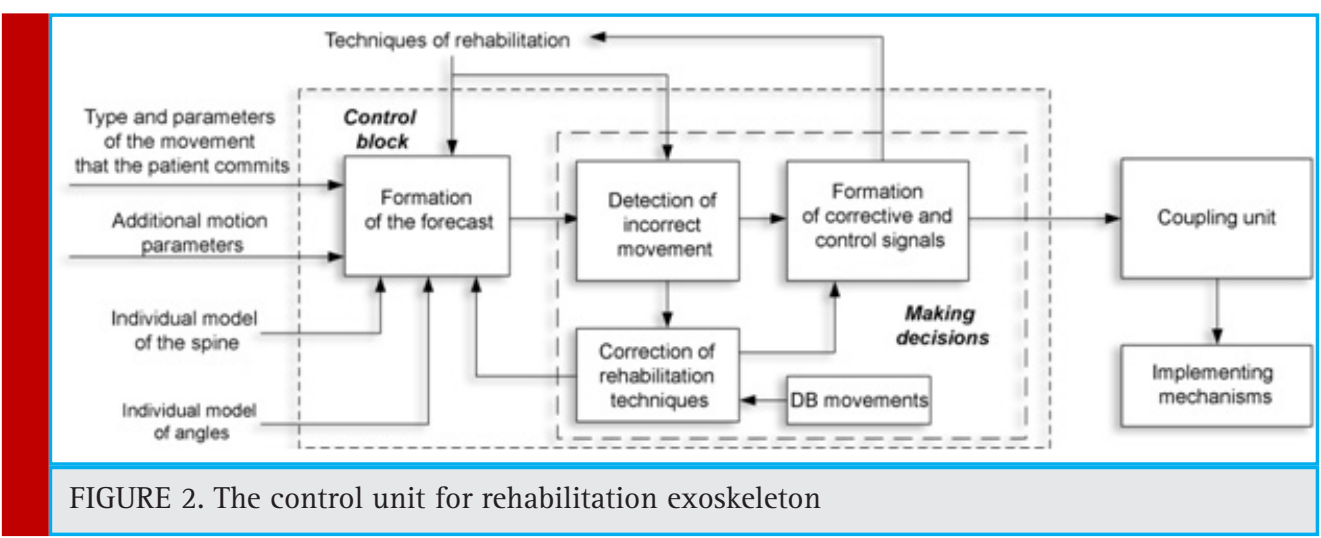

basis of the information angular model of the patient, characterizing the permissible deviations in patient movements, the individual spine model describing the geometric and spatial parameters of the main parts of the spine, the data on the movement (type, speed, angles and EMG and EEG data). The performed movements are performed in accordance with the rehabilitation technique. The algorithm of the control unit is shown in Figure 3.

A lot of work has been devoted to the main dependencies of the behavior of the musculoskeletal system, joints and their connecting components, pain sensations and thresholds of perception of pain, for example (Pezhovic et al., 2003; Pinchuk et al., 2008; Shilko \&t Ermakov,
2008; Suslov et al., 2008; Babchina et al., 2017; Grecheneva et al., 2016; Grecheneva et al., 2017). Formation of prognostic estimates of damage to the spinal sections during motion on the basis of individual models and parameters of movements are given in (Dorofeev et al., 2017).

The vector of predictive estimates for each type of tissue is described by the vector $\mathrm{F}=\{\mathrm{L}(\mathrm{X}, \mathrm{Y}, \mathrm{Z}), \mathrm{P}(\mathrm{X}, \mathrm{Y}, \mathrm{Z})\}$, where $\mathrm{L}$ is the three-dimensional vector for estimating the degree of damage, and $\mathrm{P}$ is the damage probability. The change in the projection vector in time $\mathrm{F}(\mathrm{t})$ is used in conjunction with the vector $\mathrm{M}(\mathrm{t})$, describing the rehabilitation technique (rehabilitation exercise). The vector $\mathrm{M}(\mathrm{t})=\{\mathrm{K}(\mathrm{t}), \mathrm{C}(\mathrm{t})\}$ characterizes the space-time change in

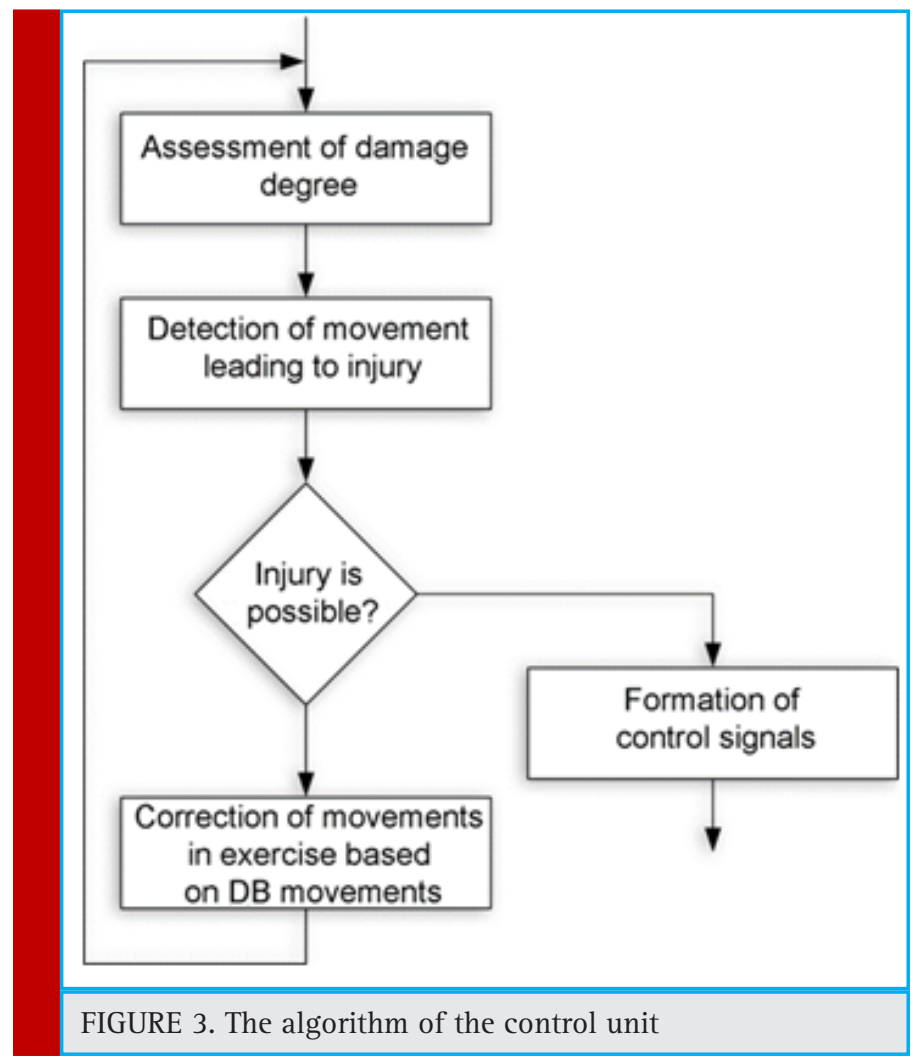




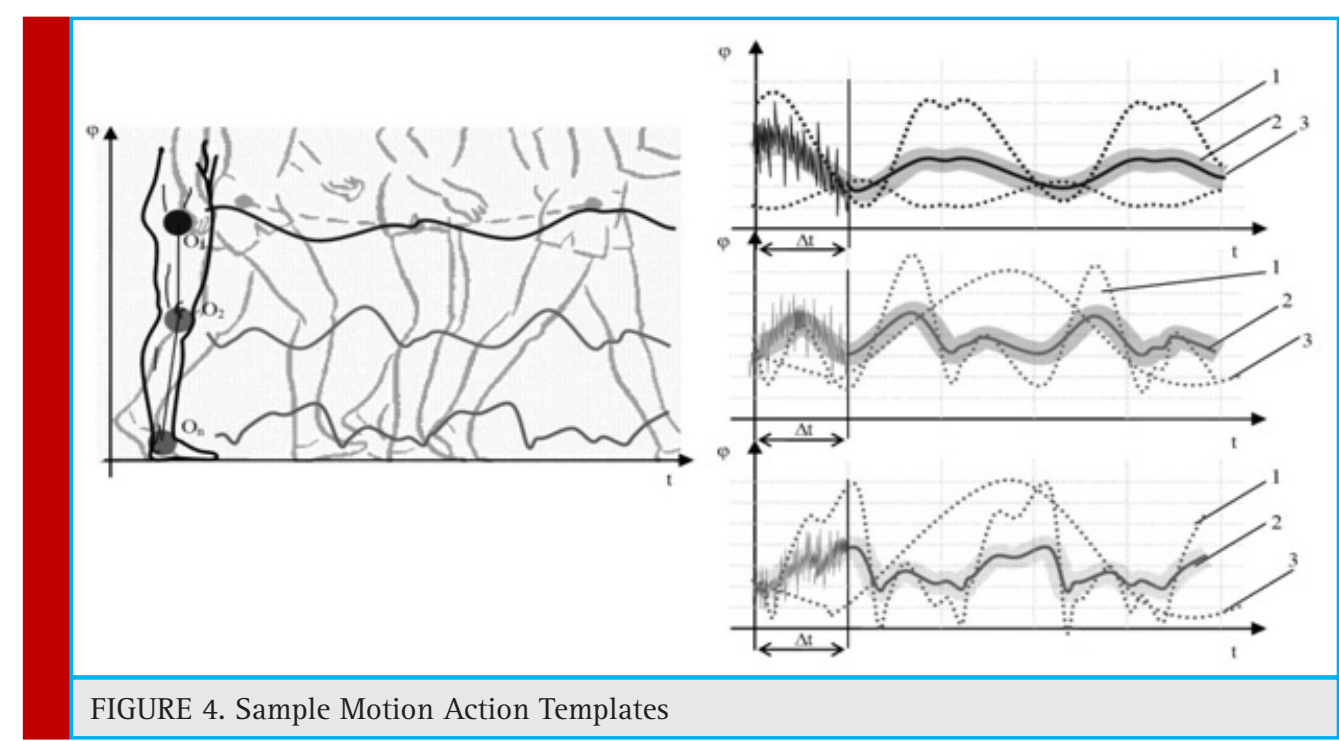

the position of the kinematic pairs of the spine $\mathrm{K}(\mathrm{t})$ and the space-time characteristics of the motion (tempo, frequency, etc.) $C(\mathrm{t})$.

Classification of movements occurs on the basis of pre-formed patterns of motor actions (Figure 4). The motion pattern can be described by the vector

$$
\vec{T}(t)=\left\{\vec{T}_{D}(t), \vec{I} b(t), \vec{U} n(t), \vec{P} s t, \vec{P} p t\right\}
$$

where $\vec{T}_{D}(t)$ is the vector of spatial change in the position of the kinematic pairs; $\overrightarrow{I b}(t), \vec{U} n(t)$ are the vectors describing the change in electrophysiological parameters during the time of motion (some examples are presented in [16]); $\vec{P} s t$ is the vector describing the spectral-temporal characteristics (frequency, power spectra, etc.) of patterns of goniometric and electrophysiological signals; $\vec{P} p t$ is the vector describing the space-time characteristics of the motion (tempo, amplitude, speed, acceleration, etc.).

Motion patterns are stored in a database, supplemented for individual characteristics and various pathologies. Database updates are necessary for automatic learning and retraining of the neural network.

When implementing the system of direct control of the exoskeleton, it is necessary to have information about the magnitude and position of the goniometric vectors and vectors of the stator and exoskeleton rotor linkage, which are measured by means of various sensors. Unlike vector systems, the direct torque control system uses only current and voltage sensors and does not require the use of a speed sensor. However, accurate estimation of the position of the flux-linkage vector of each of the exoskeleton servo drives is problematic, therefore, state observers are often used to determine the flux linkage. In the case of a medical rehabilitation exoskeleton, the patient's angular model is an observer of the state, the input of which is measured goniometric data, the components of the servo vector of the state, and an output of the general state vector is output. As an observer, it is proposed to use an artificial neural network (Fig. 5).

It is assumed that combining the method of direct torque control and neural network technologies will sig-

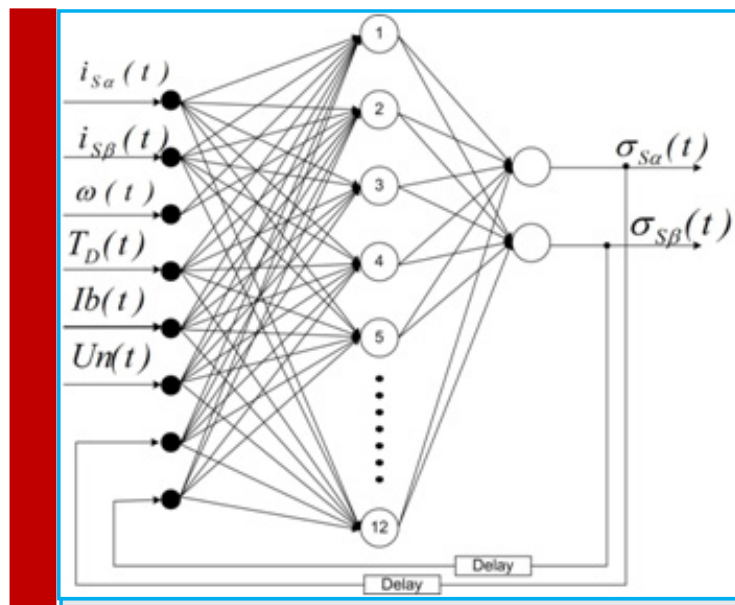

FIGURE 5. The structure of the neural network. Input signals of such a network are stator current signals $i_{S \alpha} i_{S \beta}$ of the i-node of the exoskeleton, $\omega$ the rotor frequency, $\vec{T}_{D}(t)$ is the vector of spatial change in the position of the kinematic pairs; $\overrightarrow{l b}$ $(t), \vec{U} n(t)$ are the vectors describing the change in electrophysiological parameters during the time of motion and feedback c from the neural network output delayed by one step of training (Delay block), and output signals - signals that determine the mode of operation of the exoskeleton nodes $\sigma_{S \alpha,} \sigma_{S \alpha}$. 
nificantly improve the quality of control of an asynchronous traction electric drive, as well as the robustness of the control system (resistance to changes in the parameters of the control object), thereby improving the quality of control and identification.

Simulation of the operation of the control unit was performed on the CT of patient data, which has a curvature of the cervical spine. When the head was tilted to an angle of more than 59 degrees on average, the patient experienced pain. In $87 \%$ of cases, the head inclinations were accompanied by a slight crunch in the cervical region.

Initial exercises for modeling the operation of the control unit included the inclination of the head by 90 degrees. As a result of the operation of the control unit, the initial exercises were adjusted, the maximum inclination of the head was 64 degrees (Figure 6).

\section{CONCLUSION}

Thus, the developed algorithms of the control unit allow to correct motor exercises in the rehabilitation technique for the physiological characteristics of the patient, and also do not allow the executive mechanisms to make movements dangerous to health. It should be noted that the permissible limits for the search for optimal exercises of the control unit are set by the expert and for automated work should be automatically determined from the CT data and the simulation results. These boundaries in the example under consideration were set rigidly, which was the reason for the discrepancy between the results of modeling and pain sensations of the patient being studied.

\section{ACKNOWLEDGEMENTS}

The work was supported by RFBR grant No. 16-08$00992 \_a$

\section{REFERENCES}

Babchina PI, Petrova EM, Chesnokov IV. (2017). Three-dimensional modeling in the development of a biomechanical method for determining the permissible loads on the deformed segment of the thoracic spine. Innovative materials and technologies in design. P. 37-4526

Butukhanov VV. (2009). The state of the muscular system in patients with scoliotic deformation of i-ii degree in ontogenesis. Communication II. Tremor of muscle tension in the back muscles of sciosis patients of I-II degree in different age periods. Acta Biomedica Scientifica. No. 3.

Dorofeev N, Grecheneva A, Kuzichkin 0, Romanov R. (2017). The method of the estimation of the acceptable degree of the deflection of the spine and of bias of vertebrae in systems of the diagnostic and the rehabilitation. International Multidisciplinary Scientific GeoConference Surveying Geology and Mining Ecology Management, SGEM, Volume 17, Issue 61, Pp. 1161-1168.

Doronin BM, Doronina OB. (2010). Some topical issues of diagnosis and treatment of pain in the back. Neurology, neuropsychiatry, psychosomatics. No. 4. P. 24-28

Efimov AP. (2012). Clinically significant parameters of gait. Traumatology and orthopedics of Russia. No. 1 (63). C, 60-65

Grecheneva A, Konstantinov I, Kuzichkin 0, Dorofeev N. (2017). Decision support system for real-Time diagnosis of musculoskeletal system. CEUR Workshop Proceedings. Volume 1839, Pp. 84-97

Grecheneva A, Konstantinov I, Kuzichkin O. (2016). The structure of the information system to support the goniometric accelerometric control of human biomechanics. Nano, bio and green - technologies for a sustainable future conference proceedings, sgem 2016, vol 1 International Multidisciplinary Scientific geoconference-SGEM. pp. 829-836

Grecheneva A, Kuzichkin 0, Dorofeev N. (2017). The algorithm for express-analysis of the human functional systems. International Multidisciplinary Scientific GeoConference Surveying Geology and Mining Ecology Management, Vol 17, Issue 61. 1081-1088

Kulik AA. (2017). Mathematical modeling of the movement of the bioelectric hip prosthesis. Bulletin of the Astrakhan State Technical University. Series: Management, Computer Science and Informatics. No. 2. P. 7-13

Maksimova YuA. (2012). Prevention of functional disorders of the spine by eliminating postnagruzochnyh changes in the musculoskeletal apparatus of upper acrobats. Pedagogy, psychology and medico-biological problems of physical education and sport. 12. P. 75-79

Pezhovic S, Chotek S, Filipyak J, Begzhinsky R,Yarmundovich V, Charek I. (2003). The effect of stabilization on the changes in stiffness characteristics of the cervical spine (experimental study). Russian Journal of Biomechanics. T. 7. No. 3. P. 52-60

Pinchuk LS, Chernyakova YuM, Goldede VA. (2008). Tribology of joints and problems of modern orthopedics. Friction and wear. T. 29. No. 3. P. 293-305

Rakhmilevich AB, Chantsev AV, Raspopova EA, Kolomiets AA. (2012). The diagnostic value of tribological study of the knee joint. Genius of orthopedics. No. 2. Pp. 102-105.

Shanitsin IN, Ivanov AN, Bazhanov SP, Ulyanov VYu, Norkin IA, Ninel VG, Puchin'yan DM. (2013). Methods of studying measurements in the spinal cord with traumatic injuries of the peripheral nerve. Regional circulation and microcirculation. No. 1 (49). Pp. 13-22

Shchenyavskaya EV, Zakharova TV. (2015). Method of synchronization of signals of magnetoencephalograms and myograms. Systems and Means of Informatics. Vol. 25. 4. P. 101-113

Shilko SV, Ermakov SF. (2008). The role of the liquid phase and porous structure of cartilage in the formation of biomechanical 
properties of joints. Part 1. Russian Journal of Biomechanics. 12. No. 2. P. 31-40

Sinitskaya EYu, Gribanov AV. (2014). Brain potentials associated with motion. Human Ecology. 1. P. 49-59

Sobolev VI, Popov MN, Anyukhina AV. (2017). The method of circular training as an effective way to correct the spinal column flexibility in children of primary school age. Problems of Modern Pedagogical Education. No. 54. P. 227-238

Sufianova GZ, Shapkin AG. (2014). Damage to the nervous tissue. Mechanisms, models, methods of estimation. M.: Publishing House of RAMS. P. 288

Suslov AA, Ermakov SF, Beletsky AV, Shilko SV, Nikolaev VI. (2008). The role of the liquid phase and porous structure of cartilage in the formation of biomechanical properties of joints. Part 2. Russian Journal of Biomechanics. 12. No. 4. P. 33-39

Tuktamyshev VS, Bezmaternykh VV. (2014). Modeling the influence of intra-abdominal pressure on the loading of the spinal column. Modern problems of science and education. 3 . P. 704-709

Vengerova NN, Solovyova I0. (2008). Influence of training loads on the spine of gymnasts-artists. Health - the basis of human potential: problems and ways to solve them. T. 3.1. S. 45-47
Voznesenskaya TG. (2006). Back pain: neurologist's view. Consilium Medicum. 8. No. 2. P. 75-79

Yezhov MYu, Berendeev NN, Petrov SV. (2013). Mathematical model of the development of changes in articular tissues with different intensity of physical activity. Fundamental issledvoaniya. No. 7. S. 550-554

Zakharova TV, Karpov PI, Bugaevsky V.M. (2016). Localization of the source of activity in solving the inverse problem of magnetoencephalography // Applied Mathematics and Informatics. Proceedings of the Faculty of the IOM Faculty of Moscow State University Lomonosov Moscow State University. Moscow. 12-25

Zakharova TV, Nikiforov SYu, Goncharenko MB, Dranishniki MA, Klimov GA, Khaziakhmetov MSh, Chayanov NV. (2012). Methods of Signal Processing for Localization of Irreplaceable Areas of the Brain. Systems and Means of Informatics. Vol. 22. No. 2. P. $157-175$

Zakharova TV, Shemirova AA. (2016). Methods of finding the reference points of cyclic non-stationary signals. Bulletin of Tver State University. Series: Applied Mathematics. No 3. P. 47-58

Zubareva EA. (2011). Determination of the functional condition of joints in horses in conditions of training and testing. Omsk Scientific Bulletin. No. 1 104. Pp. 165-169 